\title{
Theory of multiphoton ionization tested on negative ions
}

\author{
Theodoros Mercouris and Cleanthes A. Nicolaides \\ Theoretical and Physical Chemistry Institute, National Hellenic Research Foundation, 48 Vassileos Constantinou Avenue, \\ 11635 Athens, Greece \\ (Received 15 July 1991)
}

In a recently published perturbation theory study of multiphoton-ionization cross sections of $\mathbf{H}^{-}$[S. Geltman, Phys. Rev. A 43, 4930 (1991)], a serious discrepancy was reported between the model-based results and previous $a b$ initio Floquet-theorem-based coordinate rotation results [M. Crance, J. Phys. B 23, L285 (1990)]. We present two-and three-photon ionization cross sections from many-electron, many-photon-theory nonperturbative calculations, which essentially agree with Geltman's results. This finding supports our earlier conclusions on the physics of photodetachment without or with an external static field.

PACS number(s): $32.80 . \mathrm{Rm}, 32.80 . \mathrm{Fb}$

Because of their empty singlet excited spectrum, stable atomic negative ions are suitable for testing nonresonant multiphoton-ionization theory and for gaining insight into the physics of laser-atom interactions. Furthermore, their small ionization potentials make them attractive candidates for accurate laser- or static-field-inducedionization experiments [1-5].

For practical reasons, the theoretical treatment of the multiphoton ionization of negative ions has been approximated drastically by adopting models of short-range potentials seen by the extra electron (e.g., [6-14]). In recent years, advances in the theory led to the incorporation of the electronic structure and many-electron effects in conjunction with multiphoton or dc-field-atom interactions to different levels of accuracy [15-22]. In our two publications of $\mathrm{Li}^{-}$[19] and on $\mathrm{H}^{-}$[20], we pointed out that a discrepancy existed in previous results between different types of model-based theory $[9,10]$ and $a b$ initio theory [15-17], and that it is the former that is correct. We con-

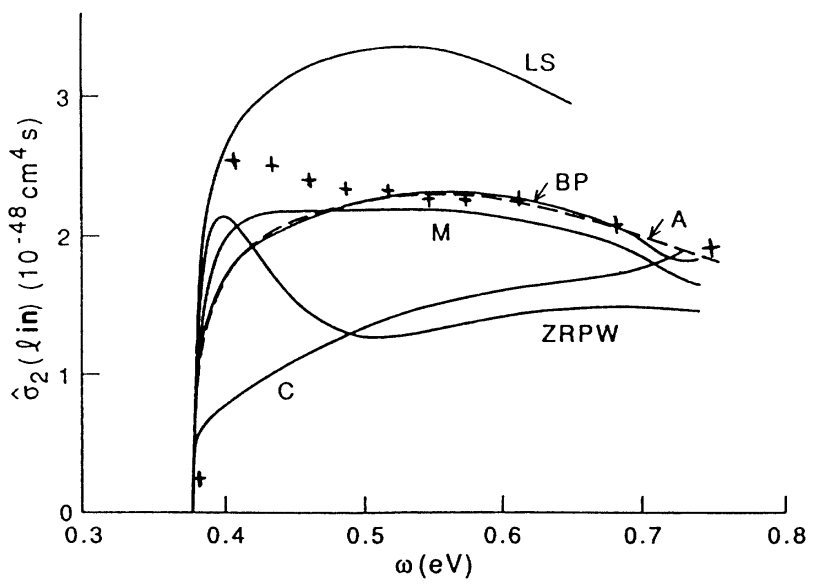

FIG. 1. Two-photon detachment cross sections as a function of laser field frequency $\omega$. This figure is taken from Fig. 4 of Geltman [14] with our results added (crosses). The initials are those used by Geltman [14]. They correspond to (C), Ref. [21]; (A), Ref. [7]; (LS), Ref. [24]; (best phase BP, model M, zerorange plane-wave ZRPW), Ref. [14]. cluded [19] that "much of the essential physics of negative ions in strong external ac and dc fields is caused by final-state effects" and these, apparently, were computed fairly accurately in the previous model-based calculations $[9,10]$. In other words, any reasonable theory that would incorporate a good representation of the perturbed final state should provide, regardless of the formalism, at least semiquantitatively accurate cross sections for negative ions.

In two recent publications on the multiphoton ionization of $\mathrm{H}^{-}$, Geltman [14] employed perturbation theory in conjunction with model-based approximations. His results for linear polarization are reproduced here. (His Figs. 4 and 7 are our Figs. 1 and 2 with our own results added.)

When comparing his results to previous theories, such as the $a b$ initio complex-coordinate rotation calculations of Crance [21], Geltman discovered a discrepancy of a factor of 2-3 for his best approximation. He concluded "we feel that the full correlation effects in the intermediate and final continuum states are taken into account in

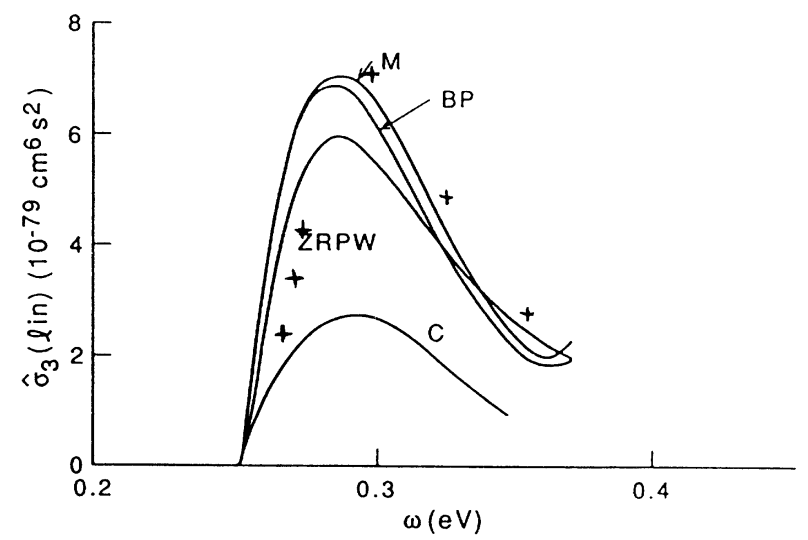

FIG. 2. Three-photon detachment cross sections as a function of laser field frequency $\omega$. This figure is taken from Fig. 7 of Geltman [14], with our results added (crosses). The initials are those used by Geltman [14]. They correspond to (C), Ref. [21]; (BP, M, ZRPW), Ref. [14]. 
our best phase results, so we do not understand the reason for such a large difference with Crance's results." He observed similar discrepancies when comparing the results with other $a b$ initio calculations as well (e.g., [16]).

Since the field of advanced multiphoton-ionization theory and calculation is relatively new, it is important to document all the available information in order to increase our understanding. Thus, we have turned to our many-electron, many-photon theory (MEMPT) and analysis already in the literature $[19,20,22]$ and have plotted our results for the two- and three-photon ionization cross sections [20] on the figures of Geltman [14]. As can be seen from our Figs. 1 and 2, the results of the MEMPT are in good agreement with those of Geltman. This fact confirms our previous conclusion [19] that in the theory of the ionization phenomena that occur from ac- or dcfield-negative-ion interactions, the important element is the accurate representation of the perturbed final state. Furthermore, it sheds additional light on a recent discussion [21-23] on the identification and method of computation of the effects of electron correlation on multiphoton ionization by strong fields.
[1] J. L. Hall, E. J. Robinson, and L. M. Branscomb, Phys. Rev. Lett. 14, 1013 (1965).

[2] H. C. Bryant, A. Mohagheghi, J. E. Stewart, J. B. Donahue, C. R. Quick, R. A. Reeder, V. Yuan, C. R. Humener, W. W. Smith, S. Cohen, W. P. Reinhardt, and L. Overman, Phys. Rev. Lett. 58, 2412 (1987).

[3] C. Blondel, R. J. Champeau, A. Crubellier, C. Delsart, H. T. Duong, and D. Marinescu, Europhys. Lett. 4, 1267 (1987).

[4] R. Trainham, G. D. Fletcher, and D. J. Larson, J. Phys. B 20, L777 (1987).

[5] C. Y. Tang, P. G. Harris, A. H. Mohagheghi, H. C. Bryant, C. R. Quick, J. B. Donahue, R. A. Reeder, S. Cohen, W. W. Smith, and J. E. Stewart, Phys. Rev. A 39, 6068 (1989).

[6] E. J. Robinson and S. Geltman, Phys. Rev. 153, 4 (1967).

[7] S. A. Adelman, J. Phys. B 6, 1986 (1973).

[8] H. R. Reiss, Phys. Rev. A 22, 1786 (1980).

[9] P. A. Golovinskii and B. A. Zon, Bull. Acad. Sci. USSR Phys. Ser. 45, 12 (1981).

[10] N. B. Delone, Yu. I. Kiyan, V. P. Krainov, and V. I. Tugushev, Opt. Spektrosk. 58, 262 (1985) [Opt. Spectros. (USSR) 58, 157 (1985)].
[11] R. Shakeshaft and X. Tang, Phys. Rev. A 36, 3193 (1987).

[12] W. G. Greenwood and J. H. Eberly, Phys. Rev. A 43, 525 (1991).

[13] J. N. Bardsley and M. J. Comella, Phys. Rev. A 39, 2252 (1989).

[14] S. Geltman, Phys. Rev. A 42, 6958 (1990); 43, 4930 (1991).

[15] M. Crance and M. Aymar, J. Phys. B 18, 3529 (1985).

[16] M. G. J. Fink and P. Zoller, J. Phys. B 18, L373 (1985).

[17] T. F. Jiang and A. F. Starace, Phys. Rev. A 38, 2347 (1988).

[18] Th. Mercouris and C. A. Nicolaides, J. Phys. B 21, L285 (1988).

[19] C. A. Nicolaides and Th. Mercouris, Chem. Phys. Lett. 159, 45 (1989).

[20] Th. Mercouris and C. A. Nicolaides, J. Phys. B 23, 2037 (1990).

[21] M. Crance, J. Phys. B 23, L285 (1990).

[22] Th. Mercouris and C. A. Nicolaides, J. Phys. B 24, L165 (1991).

[23] M. Crance, J. Phys. B 24, L169 (1991).

[24] C.-R. Lin and A. F. Starace, private communication to Geltman (Ref. [14]). 